INPLASY

PROTOCOL

To cite: Li et al. Effect of acupuncture treatment on cognitive impairment after traumatic brain injury in adults: A systematic review protocol. Inplasy protocol 2021110113. doi:

10.37766/inplasy2021.11.0113

Received: 30 November 2021

Published: 30 November 2021

Corresponding author:

$\mathrm{Na} \mathrm{Li}$

2061016@sntcm.edu.cn

Author Affiliation:

Shaanxi University of

Traditional Chinese Medicine.

Support: None.

Review Stage at time of this submission: Piloting of the study selection process.

Conflicts of interest:

None declared.

\section{Effect of acupuncture treatment on cognitive impairment after traumatic brain injury in adults: A systematic review protocol}

\author{
Li, N1; Ai, X2; Guo, X33 Liu, J4; Zhang, R5; Wang, R6.
}

Review question / Objective: Are acupuncture more effective than control interventions (i.e. treatment as sham acupuncture or placebo) in the treatment of motor and cognitive impairment after traumatic brain injury in adults? Information sources: search database:The following electronic databases will be searched for relevant literature: the Cochrane Library, MEDLINE, EMBASE, Web of Science, Springer, the Chinese Science Citation Database (CSCD), China National Knowledge Infrastructure (CNKI), the Chinese Biomedical Literature Database (CBM),Wanfang, and. the Chinese Scientific Journal Database (VIP). Time limit: the searches will be conducted from the inception of each database to November 30, 2021. Protocol of Systematic review and Meta analysis of acupuncture in the treatment of cognitive impairment after traumatic brain injury and the included literatures were all RCTS with English and Chinese on language.

INPLASY registration number: This protocol was registered with the International Platform of Registered Systematic Review and Meta-Analysis Protocols (INPLASY) on 30 November 2021 and was last updated on 30 November 2021 (registration number INPLASY2021110113).

\section{INTRODUCTION}

Review question / Objective: Are acupuncture more effective than control interventions (i.e. treatment as sham acupuncture or placebo) in the treatment of motor and cognitive impairment after traumatic brain injury in adults?
Rationale: TBI is a significant public health concern and a leading cause of disability in the global world that impacts over 50 million people annually. With the continuous progress of social industrialization, the incidence of TBI has been increasing. The majority of TBls are from road traffic accidents, falls, injuries in the workplace and assaults. TBI is 
associated with long-term disability, which can significantly impact daily functioning and quality of life. The sequelae following TBI often includes physical and cognitive difficulties. Cognitive symptoms include deficits in attention, executive functioning, memory (encoding and retrieval), language, visuoperceptual functioning, and psychomotor functioning abilities. Acupuncture is a treatment modality originating from traditional Chinese medicine and has been used to treat various cognitive impairment diseases and clinical trials have studied its application in brain injury.

Condition being studied: Two reviewers (LN, AX) will select the included studies and will extract relevant data independently from the studies. In case of disagreement, a third party shall be consulted to assist judgment, and the missing information shall be supplemented by contacting the author. In the literature selection, the title and abstract of the literature were first read through NoteExpress. After excluding obviously overlapping papers, the full text was further read to determine whether to include them in the end. According to the title and abstract, the literatures that obviously did not meet the inclusion requirements were screened out, and the literatures that might meet the requirements were downloaded and read in full to further determine whether they met the inclusion criteria. And the extraction content included extraction of data according to the title, author, year of publication, number of papers, number of cases, intervention measures, outcome indicators.

\section{METHODS}

Search strategy: Search strategy used in MEDLINE database \#1 Title/Abstract: Brain Injury, traumatic \#2 Title/Abstract: Traumatic brain injuries \#3 Title/Abstract: Traumatic brain injury \#4 Title/Abstract: TBI \#5 Title/Abstract: Brain trauma \#6 Title/ Abstract: Brain traumas \#7 Title/Abstract: Traumas, brain \#8 Title/Abstract: Trauma, brain \#9 Title/Abstract: Encephalopathy, traumatic \#10 Title/Abstract: Traumatic encephalopathy \#11 Title/Abstract: Head injury \#12 Title/Abstract: Closed head injury \#13 Title/Abstract: Closed head injuries \#14 Title/Abstract: Head injury, closed \#15 Title/ Abstract: Head trauma \#16 Title/Abstract: Head injury, nonpenetrating \#17 Title/ Abstract: Head injuries, nonpenetrating \#18 Title/Abstract: Blunt head injury \#19 Title/ Abstract: Blunt head injuries \#20 Title/ Abstract: Head injuries, blunt \#21 Title/ Abstract: Prefrontal cortex damage \#22 OR 1-21 \#23 Title/Abstract: Cognitive Dysfunctions \#24 Title/Abstract: Dysfunction, Cognitive \#25 Title/Abstract: Dysfunctions, Cognitive \#26 Title/Abstract: Cognitive Impairments \#27 Title/Abstract: Cognitive Impairment \#28 Title/Abstract: Impairment, Cognitive \#29 Title/Abstract: Impairments, Cognitive \#30 Title/Abstract: Mild Cognitive Impairment \#31 Title/ Abstract: Cognitive Impairment, Mild \#32 Title/Abstract: Cognitive Impairments, Mild \#33 Title/Abstract: Impairment, Mild Cognitive \#34 Title/Abstract: Impairments, Mild Cognitive \#35 Title/Abstract: Mild Cognitive Impairments \#36 Title/Abstract: Mild Neurocognitive Disorder \#37 Title/ Abstract: Disorder, Mild Neurocognitive \#38 Title/Abstract: Disorders, Mild Neurocognitive \#39 Title/Abstract: Mild Neurocognitive Disorders \#40 Title/ Abstract: Neurocognitive Disorder, Mild \#41 Title/Abstract: Neurocognitive Disorders, Mild \#42 Title/Abstract: Cognitive Decline \#43 Title/Abstract: Cognitive Declines \#44 Title/Abstract: Decline, Cognitive \#45 Title/ Abstract: Declines, Cognitive \#46 Title/ Abstract: Mental Deterioration \#47 Title/ Abstract: Deterioration, Mental \#48 Title/ Abstract: Deteriorations, Mental \#49 Title/ Abstract: Mental Deteriorations \#50 OR 23-49 \#51 Title/Abstract:Acupuncture therapy \#52 Title/Abstract:Acupuncture therapy \#53 Title/Abstract: Pharmacoacupuncture treatment \#54 Title/ Abstract: Pharmacoacupuncture therapy \#55 Title/Abstract: Acupuncture \#56 Title/ Abstract: Acupoints \#57 Title/Abstract: Acupunct \#58 Title/Abstract: Manual acupuncture \#59 Title/Abstract: Body acupuncture \#60 Title/Abstract: Scalp acupuncture \#61 Title/Abstract: Auricular acupuncture \#62 Title/Abstract: Auriculotherapies \#63 Title/Abstract: 
Electroacupuncture \#64 Title/Abstract:Fire needling \#65 Title/Abstract: Warm needling \#66 Title/Abstract: Elongated needle \#67 Title/Abstract: Intradermal needling \#68 Title/Abstract: Dermal needle \#69 Title/ Abstract: Plum blossom needle \#70 OR 51-69 \#71 Title/Abstract: randomized controlled trial \#72 Title/Abstract: controlled clinical trial \#73 Title/Abstract: randomized. \#74 Title/Abstract: randomly \#75 Title/Abstract: RCT \#76 Title/Abstract: trial \#77 OR 71-76 \#78 Title/Abstract: $\geq 18$ years of age \#79 Human \#80 \#22 AND \#50 AND \#70 AND \#77AND\#78 AND\#79.

Participant or population: Adults (18 years old and over) diagnosed as having cognitive impairment after traumatic brain injury according to Montreal cognitive assessment scale (MoCA) criteria will be included with no restrictions on gender, race, nationality and medical units.

Intervention: Experimental group: acupuncture or acupuncture combined with other treatments.

Comparator: Trials will be included if they include any of the following control groups:- Sham acupuncture;• Placebo;• No treatment; $\cdot$ other active therapies;' acupuncture in addition to active therapy compared with the same active therapy.We will exclude RCTs in which one form of acupuncture has only been compared with another form of acupuncture, or a different type of TCM (e.g., Chinese herbal medicine).

Study designs to be included: We will include randomised controlled trials only. These literatures will be excluded: Quasirandomized RCT; duplicated literature; No literature was found; not acupuncture, not cognitive impairment of TBI, not RCT.

\section{Eligibility criteria: (1) Montreal cognitive} assessment scale (MoCA) < 26. (2) Men or women aged $\geq 18$ years old (3) The patients sign informed consent and cooperate with the treatment
Information sources: search database:The following electronic databases will be searched for relevant literature: the Cochrane Library, MEDLINE, EMBASE, Web of Science, Springer, the Chinese Science Citation Database (CSCD), China National Knowledge Infrastructure (CNKI), the Chinese Biomedical Literature Database (CBM),Wanfang, and. the Chinese Scientific Journal Database (VIP). Time limit: the searches will be conducted from the inception of each database to November 30, 2021. Protocol of Systematic review and Meta analysis of acupuncture in the treatment of cognitive impairment after traumatic brain injury and the included literatures were all RCTS with English and Chinese on language.

Main outcome(s): Montreal cognitive assessment scale(MoCA).

Additional outcome(s): Mini-mental State Examination (MMSE); Barthel index(BI).

Quality assessment / Risk of bias analysis: The risk of bias will be assessed using the Cochrane Collaboration's tool for randomized trials.

Strategy of data synthesis: A narrative (descriptive) synthesis is planned if the outcomes lack of homogeneity (or there is very little), such that they cannot be pooled in RevMan. A quantitative synthesis will, however, be used if the included studies are found to be sufficiently homogenous.

Subgroup analysis: We will pool trials if the interventions of the studies is similar, and specific subgroups according to the similar intervention forms or designs will be analyzed.

Sensitivity analysis: We will perform sensitivity analyses by excluding:studies with high risks of bias and outliers that are numerically distant from the rest of the data.

Language: English.

Country(ies) involved: China. 
Keywords: Acupuncture, traumatic brain injury, cognitive impairment, randomized controlled trials, systematic review, protocol.

Contributions of each author:

Author 1 - Ms Na Ll. Chengdu university of traditional chinese medicine,Chengdu, sichuan province, Shaanxi university of traditional Chinese medicine,Xi 'an, shaanxi province

Author 2 - Ms Xia Al. Shaanxi university of traditional Chinese medicine,,Xi 'an, shaanxi province

Author 3 - Mr Xinrong Guo. Shaanxi university of traditional Chinese medicine,,Xi 'an, shaanxi province

Author 4 - Ms Juan Liu. Shaanxi university of traditional Chinese medicine,,Xi 'an, shaanxi province

Author 5 - Mr Rongchao Zhang. Shaanxi university of traditional Chinese medicine,, $X i$ 'an, shaanxi province Professor

Author 6 - Ruihui Wang. Shaanxi university of traditional Chinese medicine,, Xi 'an, shaanxi province. 